\title{
ON THE CONTROL OF HEAT CONDUCTION
}

\author{
FAYZIEV YUSUf ERGASHEVICH \\ National University of Uzbekistan, \\ 700174, Students Town, Tashkent, Uzbekistan. \\ Corresponding author: fayziev.yusuf@mail.ru \\ (Received: th $^{\text {th }}$ Feb 2017; Accepted: $1^{\text {th }}$ March 2018; Published on-line: $1^{\text {st }}$ June 2018) \\ https://doi.org/10.31436/iiumej.v19i1.796
}

\begin{abstract}
Mathematical models of thermo control processes in a rectangular plate are considered. In the model under consideration, the temperature inside a plate is controlled by heat exchange through one boundary while the other three are insulated. The control parameter is a function that satisfies certain integral equations. Sufficient conditions for achieving the given projection of the temperature at a fixed point on the plate and given average temperature are studied.

ABSTRAK: Model matematik bagi proses kawalan suhu dalam bekas segi empat tepat telah dipilih. Melalui model ini, suhu bekas dikawal dengan menukar haba melalui salah satu sisi bekas, manakala tiga sisi lain telah ditebat. Parameter kawalan ini ialah fungsi, di mana ia sesuai dengan persamaan sesetengah integral. Keadaan sesuai bagi mencapai suhu tetap bekas seperti cadangan dan suhu purata yang diberikan turut dikaji.
\end{abstract}

KEYWORDS: non-homogeneous heat equation; control of heat conduction; boundary conditions; initial conditions

\section{INTRODUCTION}

Many physical processes and engineering problems are described by heat/diffusion equations and the study of the properties of the solutions is important. Methods of the solutions of various boundary value problems and problems with the initial conditions can be found in [1]. For the first time, a detailed explanation of the problems of control of the system with distributed parameters, described by partial differential equations were given in [2].

In recent years, interest in the study of a system with distributed parameters has increased significantly. In works [3-5] by Il'in and Moiseev, the questions of boundary control by the various systems described by a wave equation are studied. Notable works $[6,7]$, studied problems related to the process of control associated with the equations of parabolic type, particularly the heat transfer process.

Equation (1), in the homogeneous case, describes the problem of heat control studied by Alimov in [8-12]. The problem for the non-homogeneous equation (1), in the case of heat transfer, is a rod studied in [13].

\section{FORMULATION OF PROBLEMS}

Consider the non-homogeneous heat equation 


$$
u_{t}=a^{2}\left(u_{x x}+u_{y y}\right)+f(x, y, t), \quad 0<x<l_{1}, \quad 0<y<l_{2}, t>0
$$

with the boundary conditions

$$
\left\{\begin{array}{l}
u(0, y, t)=\mu(t), \quad u\left(l_{1}, y, t\right)=0, \\
u(x, 0, t)=0, \quad u\left(x, l_{2}, t\right)=0, \quad t \geq 0 \\
\mu(0)=0, \quad|\mu(t)| \leq M
\end{array}\right.
$$

and initial conditions

$$
u(x, y, 0)=\varphi(x, y), \quad 0 \leq x \leq l_{1}, \quad 0 \leq y \leq l_{2},
$$

where $\varphi(x, y)$ - a given function and $M-$ a positive number.

It is known that the boundary condition (2) means $\mu(t)$ heat entry from the $x=0$ side of the rectangle and zero temperature kept in other sides. In the present paper we study following problems:

Problem 1: Let $B>0$ be given number. It is required to find such a temperature $\mu(t)$ that problems (1) - (3) has a solution satisfying following integral relation (Fig. 1):

$$
\int_{0}^{l_{1}} \int_{0}^{l_{2}} u(x, y, t) d y d x=B
$$

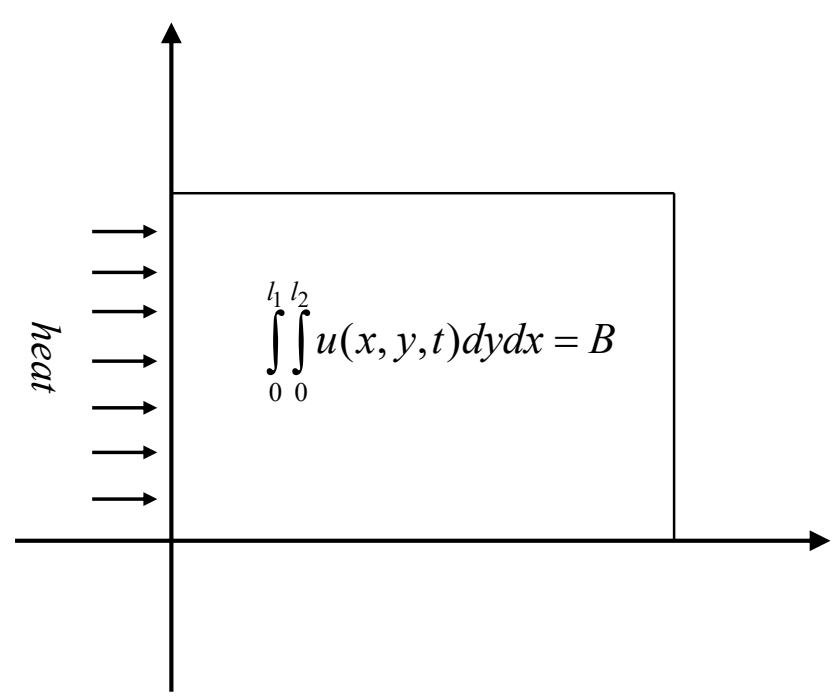

Fig. 1: Total heat.

Problem 2: Let $B>0$ be a given number. Find a temperature $\mu(t)$ such that the problem (1) - (3) has a solution that at time $t$ at inside point $A\left(x_{o}, y_{o}\right)$ satisfies the condition (Fig. 2):

$$
u\left(x_{o}, y_{o}, t\right)=B
$$




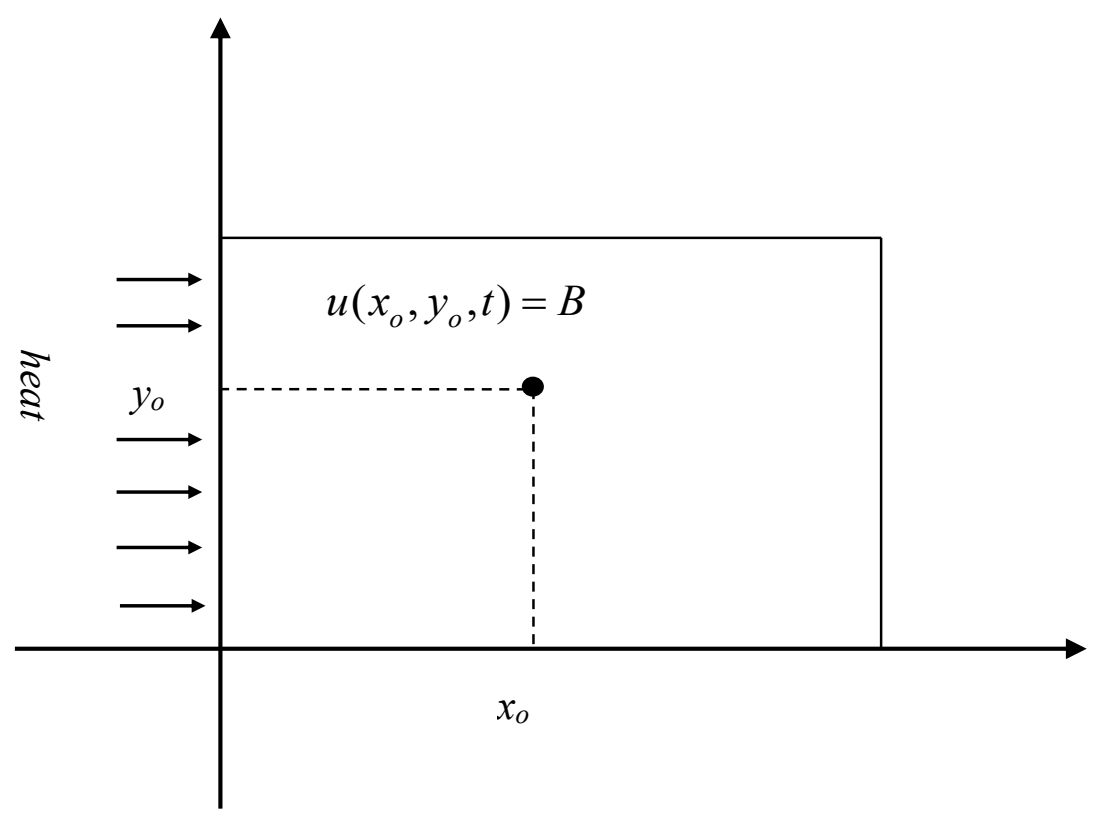

Fig. 2: The temperature at point $\left(x_{o}, y_{o}\right)$.

Problem 3: Now we consider equation (1) with the initial condition (3) and the boundary conditions as follows

$$
\left\{\begin{array}{l}
u(0, y, t)=0, \quad u\left(l_{1}, y, t\right)=\mu(t), \\
u(x, 0, t)=0, \quad u\left(x, l_{2}, t\right)=0, \\
\mu(0)=0, \quad|\mu(t)| \leq M
\end{array} \quad t \geq 0\right.
$$

Let $B>0$ be a given number. Find a temperature $\mu(t)$ such that problems (1), (3), (6) have a solution that satisfies (4).

Note, that boundary condition (6) means that heat flow $\mu(t)$ is coming from the boundary $x=l_{1}$ other boundaries kept with zero temperature.

\section{THE SOLUTION OF PROBLEM 1}

In this section we solve problem 1 .

Theorem 1: Let $B>0$ be given number. At the time $t$ a solution of the problem (1)(3) satisfying the condition (4) if for the function $\mu(t)$ following equality is true:

$$
\int_{0}^{t} K(t, \tau) \mu(\tau) d \tau=g(t)
$$

where

$$
K(t, \tau)=\frac{4 l_{1} l_{2}}{\pi^{4}} \sum_{n=1}^{\infty} \sum_{m=1}^{\infty} \frac{\left[1-(-1)^{n}\right]\left[1-(-1)^{m}\right]^{2}}{n^{2} m^{2}}\left(\lambda_{n m} a\right)^{2} e^{-\left(\lambda_{n m} a\right)^{2}(t-\tau)}
$$




$$
\begin{aligned}
& g(t)=B-\frac{l_{1} l_{2}}{\pi^{2}} \sum_{n=1}^{\infty} \sum_{m=1}^{\infty} \frac{\left[1-(-1)^{n}\right]\left[1-(-1)^{m}\right]}{n m}\left[\hat{f}_{n m}(t)+c_{n m}\right] e^{-\left(\lambda_{n m} a\right)^{2} t} \\
& \hat{f}_{n m}(t)=\frac{4}{l_{1} l_{2}} \int_{0}^{t} \int_{0}^{l_{1}} \int_{0}^{l_{2}} f(\xi, \eta, \tau) \sin \frac{\pi n \xi}{l_{1}} \sin \frac{\pi m \eta}{l_{2}} e^{\left(\lambda_{n m} a\right)^{2} \tau} d \eta d \xi d \tau \\
& c_{n m}=\frac{4}{l_{1} l_{2}} \int_{0}^{l_{1}} \int_{0}^{l_{2}} \varphi(\xi, \eta) \sin \frac{\pi n \xi}{l_{1}} \sin \frac{\pi m \eta}{l_{2}} d \eta d \xi \\
& \lambda_{n m}=\sqrt{\left(\frac{\pi n}{l_{1}}\right)^{2}+\left(\frac{\pi m}{l_{2}}\right)^{2}}
\end{aligned}
$$

Proof: Solution of the problem (1) - (3) can be represented as following series:

$$
u(x, y, t)=U(x, y, t)+\sum_{n=1}^{\infty} \sum_{m=1}^{\infty}\left[\bar{f}_{n m}(t)+c_{n m}\right] e^{-\left(\lambda_{n m} a\right)^{2} t} \sin \alpha_{n} x \cdot \sin \beta_{m} y,
$$

where

$$
\begin{aligned}
& U(x, y, t)=\left\{\begin{array}{ccc}
\frac{l_{1}-x}{l_{1}} \mu(t) & \text { if } \quad 0<y<l_{2} \\
0 & \text { if } \quad y=0, y=l_{2}
\end{array}\right. \\
& \bar{f}_{n m}(t)=\frac{4}{l_{1} l_{2}} \int_{0}^{t} \int_{0}^{l_{1}} \int_{0}^{l_{2}}\left[f(\xi, \eta, \tau)+\frac{\xi-l_{1}}{l_{1}} \mu^{\prime}(\tau)\right] \sin \alpha_{n} \xi \cdot \sin \beta_{m} \eta \cdot e^{\lambda_{n m}^{2} a^{2} \tau} d \eta d \xi d \tau \\
& \alpha_{n}=\frac{\pi n}{l_{1}}, \quad \beta_{m}=\frac{\pi m}{l_{2}} \text { and } c_{n m} \text { is defined by (11). }
\end{aligned}
$$

According the Theorem a solution should satisfy condition (4) which means

$$
\begin{aligned}
& B=\int_{0}^{l_{1}} \int_{0}^{l_{2}} u(x, y, t) d y d x=\int_{0}^{l_{1}} \int_{0}^{l_{2}} \frac{l_{1}-x}{l_{1}} \mu(t) d y d x+ \\
& +\int_{0}^{l_{1} \int_{0}} \sum_{n=1}^{\infty} \sum_{m=1}^{\infty}\left[\bar{f}_{n m}(t)+c_{n m}\right] e^{-\left(\lambda_{n m} a\right)^{2} t} \sin \alpha_{n} x \cdot \sin \beta_{m} y d y d x .
\end{aligned}
$$

In the second term, we change the order of integration and summation after evaluation to get

$$
B=\frac{l_{1} l_{2}}{2} \mu(t)+\frac{l_{1} l_{2}}{\pi^{2}} \sum_{n=1}^{\infty} \sum_{m=1}^{\infty}\left[\bar{f}_{n m}(t)+c_{n m}\right] e^{-\left(\lambda_{n m} a\right)^{2} t} \frac{1-(-1)^{n}}{n} \frac{1-(-1)^{m}}{m} .
$$

Then from (14) and (10) obtain 


$$
\begin{aligned}
& B=\frac{l_{1} l_{2}}{2} \mu(t)+\frac{l_{1} l_{2}}{\pi^{2}} \sum_{n=1}^{\infty} \sum_{m=1}^{\infty}\left[\hat{f}_{n m}(t)+c_{n m}\right] e^{-\left(\lambda_{n m} a\right)^{2} t} \frac{\left[1-(-1)^{n}\right]\left[1-(-1)^{m}\right]}{n m}+ \\
& +\frac{4}{\pi^{2}} \sum_{n=1}^{\infty} \sum_{m=1}^{\infty}\left[\int_{0}^{t} \int_{0}^{l_{1} l_{2}} \int_{0}^{x-l_{1}} \mu^{\prime}(\tau) e^{-\left(\lambda_{n m} a\right)^{2}(t-\tau)} \sin \alpha_{n} x \cdot \sin \beta_{m} y d y d x d \tau\right] \times \\
& \times \frac{\left[1-(-1)^{n}\right]\left[1-(-1)^{m}\right]}{n m} .
\end{aligned}
$$

Due to $\mu(0)=0$ the last integrals can be evaluated as follows

$$
\begin{aligned}
& \int_{0}^{t} \mu^{\prime}(\tau) e^{\left(\lambda_{n m} a\right)^{2} \tau} d \tau=\mu(t) e^{\left(\lambda_{n m} a\right)^{2} t}-\left(\lambda_{n m} a\right)^{2} \int_{0}^{t} \mu(\tau) e^{\left(\lambda_{n m} a\right)^{2} \tau} d \tau, \\
& \int_{0}^{l_{2}} \sin \beta_{m} y d y=\frac{l_{2}}{\pi m}\left[1-(-1)^{m}\right], \\
& \int_{0}^{l_{1}} \frac{x-l_{1}}{l_{1}} \sin \alpha_{n} x d x=-\frac{l_{1}}{\pi n} .
\end{aligned}
$$

Thus, we have

$$
\begin{aligned}
& B=\frac{l_{1} l_{2}}{2} \mu(t)+\frac{l_{1} l_{2}}{\pi^{2}} \sum_{n=1}^{\infty} \sum_{m=1}^{\infty}\left[\hat{f}_{n m}(t)+c_{n m}\right] e^{-\left(\lambda_{n m} a\right)^{2} t} \frac{\left[1-(-1)^{n}\right]\left[1-(-1)^{m}\right]}{n m}- \\
& -\frac{4 l_{1} l_{2}}{\pi^{4}} \sum_{n=1}^{\infty} \sum_{m=1}^{\infty}\left[\mu(t)-\left(\lambda_{n m} a\right)^{2} \int_{0}^{t} \mu(\tau) e^{-\left(\lambda_{n m} a\right)^{2}(t-\tau)} d \tau\right] \frac{\left[1-(-1)^{n}\right]\left[1-(-1)^{m}\right]^{2}}{n^{2} m^{2}} .
\end{aligned}
$$

Write the last equation in a form

$$
\begin{aligned}
& B=\frac{l_{1} l_{2}}{2} \mu(t)+\frac{l_{1} l_{2}}{\pi^{2}} \sum_{n=1}^{\infty} \sum_{m=1}^{\infty}\left[\hat{f}_{n m}(t)+c_{n m}\right] e^{-\left(\lambda_{n m} a\right)^{2} t} \frac{\left[1-(-1)^{n}\right]\left[1-(-1)^{m}\right]}{n m}- \\
& -\frac{4 l_{1} l_{2}}{\pi^{4}} \mu(t) \sum_{i=1}^{\infty} \sum_{j=1}^{\infty} \frac{8}{(2 i-1)^{2}(2 j-1)^{2}}+ \\
& +\int_{0}^{t} \mu(\tau)\left\{\frac{4 l_{1} l_{2}}{\pi^{4}} \sum_{n=1}^{\infty} \sum_{m=1}^{\infty} \frac{\left[1-(-1)^{n}\right]\left[1-(-1)^{m}\right]^{2}}{n^{2} m^{2}}\left(\lambda_{n m} a\right)^{2} e^{-\left(\lambda_{n m} a\right)^{2}(t-\tau)}\right\} d \tau .
\end{aligned}
$$

After evaluation of the series in third term and taking into account following notations

$$
K(t, \tau)=\frac{4 l_{1} l_{2}}{\pi^{4}} \sum_{n=1}^{\infty} \sum_{m=1}^{\infty} \frac{\left[1-(-1)^{n}\right]\left[1-(-1)^{m}\right]^{2}}{n^{2} m^{2}}\left(\lambda_{n m} a\right)^{2} e^{-\left(\lambda_{n m} a\right)^{2}(t-\tau)},
$$




$$
G(t)=\frac{l_{1} l_{2}}{\pi^{2}} \sum_{n=1}^{\infty} \sum_{m=1}^{\infty}\left[\hat{f}_{n m}(t)+c_{n m}\right] e^{-\left(\lambda_{n m} a\right)^{2} t} \frac{\left[1-(-1)^{n}\right]\left[1-(-1)^{m}\right]}{n m} .
$$

To obtain

$$
B=\frac{l_{1} l_{2}}{2} \mu(t)-\frac{32 l_{1} l_{2}}{\pi^{4}} \mu(t) \cdot \frac{\pi^{2}}{8} \cdot \frac{\pi^{2}}{8}+\int_{0}^{t} K(t, \tau) \mu(\tau) d \tau+G(t) .
$$

Thus we obtain the following integral equation

$$
\int_{0}^{t} K(t, \tau) \mu(\tau) d \tau=B-G(t)
$$

If we denote $g(t)=B-G(t)$ then

$$
\int_{0}^{t} K(t, \tau) \mu(\tau) d \tau=g(t)
$$

Theorem 1 is proven.

\section{THE SOLUTION OF PROBLEM 2}

In this section we study problem 2 .

Theorem 2: Let $B>0$ a given number. At time $t$ a solution of the problem (1) - (3) satisfies the condition (5) if function $\mu(t)$ satisfies following integral equation

$$
\int_{0}^{t} K\left(x_{o}, y_{o}, t, \tau\right) \mu(\tau) d \tau=g(t)
$$

where

$$
\begin{aligned}
& K\left(x_{o}, y_{o}, t, \tau\right)=\frac{4}{\pi^{2}} \sum_{n=1}^{\infty} \sum_{m=1}^{\infty} \frac{\left[1-(-1)^{m}\right]}{n m}\left[\left(\lambda_{n m} a\right)^{2}\right] e^{-\left(\lambda_{n m} a\right)^{2}(t-\tau)} \sin \alpha_{n} x_{o} \cdot \sin \beta_{m} y_{o}, \\
& g(t)=B-\sum_{n=1}^{\infty} \sum_{m=1}^{\infty}\left[\hat{f}_{n m}(t)+c_{n m}\right] e^{-\left(\lambda_{n m} a\right)^{2} t} \sin \alpha_{n} x_{o} \cdot \sin \beta_{m} y_{o} .
\end{aligned}
$$

Proof: It is known that a solution of problems (1) - (3) has the form of (13). Let this solution satisfies condition (5). Then obtain the following equality

$$
\begin{aligned}
& B=u\left(x_{o}, y_{o}, t\right)=\frac{l_{1}-x_{o}}{l_{1}} \mu(t)+ \\
& +\sum_{n=1}^{\infty} \sum_{m=1}^{\infty}\left[\bar{f}_{n m}(t)+c_{n m}\right] e^{-\left(\lambda_{n m} a\right)^{2} t} \sin \alpha_{n} x_{o} \cdot \sin \beta_{m} y_{o} .
\end{aligned}
$$

Using equality (14) obtain following 


$$
\begin{aligned}
& B=\frac{l_{1}-x_{o}}{l_{1}} \mu(t)+\sum_{n=1}^{\infty} \sum_{m=1}^{\infty}\left[\hat{f}_{n m}(t)+c_{n m}\right] e^{-\left(\lambda_{n m} a\right)^{2} t} \sin \alpha_{n} x_{o} \cdot \sin \beta_{m} y_{o}+ \\
& +\frac{4}{l_{1} l_{2}} \sum_{n=1}^{\infty} \sum_{m=1}^{\infty}\left[\int_{0}^{t} \int_{0}^{l_{1}} \int_{0}^{l_{2}} \frac{\xi-l_{1}}{l_{1}} \mu^{\prime}(\tau) e^{-\left(\lambda_{n m} a\right)^{2}(t-\tau)} \sin \alpha_{n} \xi \cdot \sin \beta_{m} \eta d \eta d \xi d \tau\right] \times \\
& \times e^{-\left(\lambda_{n m} a\right)^{2} t} \sin \alpha_{n} x_{o} \cdot \sin \beta_{m} y_{o} .
\end{aligned}
$$

As it was done in the previous problem, by evaluation of the integrals, we obtain following equality

$$
\begin{aligned}
& B=\frac{l_{1}-x_{o}}{l_{1}} \mu(t)+G\left(x_{o}, y_{o}, t\right)- \\
& -\frac{4}{\pi^{2}} \sum_{n=1}^{\infty} \sum_{m=1}^{\infty} \frac{\left[1-(-1)^{m}\right]}{n m}\left[\mu(t)-\left(\lambda_{n m} a\right)^{2} \int_{0}^{t} \mu(\tau) e^{-\left(\lambda_{n m} a\right)^{2}(t-\tau)} d \tau\right] \sin \alpha_{n} x_{o} \cdot \sin \beta_{m} y_{o}
\end{aligned}
$$

where

$$
G\left(x_{o}, y_{o}, t\right)=\sum_{n=1}^{\infty} \sum_{m=1}^{\infty}\left[\hat{f}_{n m}(t)+c_{n m}\right] e^{-\left(\lambda_{n m} a\right)^{2} t} \sin \alpha_{n} x_{o} \cdot \sin \beta_{m} y_{o} .
$$

From this we get

$$
\begin{aligned}
& B=\frac{l_{1}-x_{o}}{l_{1}} \mu(t)+G\left(x_{o}, y_{o}, t\right)- \\
& -\frac{4}{\pi^{2}} \mu(t)\left\{\sum_{n=1}^{\infty} \frac{\sin \frac{\pi x_{o} n}{l_{1}}}{n}\right\}\left\{\sum_{k=1}^{\infty} \frac{2 \sin \frac{\pi y_{o}(2 k-1)}{l_{2}}}{2 k-1}\right\}+ \\
& +\int_{0}^{t} \mu(\tau)\left[\frac{4}{\pi^{2}} \sum_{n=1}^{\infty} \sum_{m=1}^{\infty} \frac{\left[1-(-1)^{m}\right]}{n m}\left[\left(\lambda_{n m} a\right)^{2}\right] e^{-\left(\lambda_{n m} a\right)^{2}(t-\tau)} \sin \alpha_{n} x_{o} \cdot \sin \beta_{m} y_{o}\right] d \tau .
\end{aligned}
$$

By evaluation of the series in the third term and using following notation

$$
K\left(x_{o}, y_{o}, t, \tau\right)=\frac{4}{\pi^{2}} \sum_{n=1}^{\infty} \sum_{m=1}^{\infty} \frac{\left[1-(-1)^{m}\right]}{n m}\left[\left(\lambda_{n m} a\right)^{2}\right] e^{-\left(\lambda_{n m} a\right)^{2}(t-\tau)} \sin \alpha_{n} x_{o} \cdot \sin \beta_{m} y_{o}
$$

obtain

$$
\begin{aligned}
& B=\frac{l_{1}-x_{o}}{l_{1}} \mu(t)-\frac{l_{1}-x_{o}}{l_{1}} \mu(t)+\int_{0}^{t} K\left(x_{o}, y_{o}, t, \tau\right) \mu(\tau) d \tau+G\left(x_{o}, y_{o}, t\right)= \\
& =\int_{0}^{t} K\left(x_{o}, y_{o}, t, \tau\right) \mu(\tau) d \tau+G\left(x_{o}, y_{o}, t\right) .
\end{aligned}
$$


If we denote $g(t)=B-G\left(x_{o}, y_{o}, t\right)$, the obtained integral is equation (15). Theorem 2 is proven.

\section{THE SOLUTION OF PROBLEM 3}

In this section we find the solution of problem 3 .

Theorem 3: Let $B>0$ be a given number. At a time $t$, the problem (1), (3), (6) has a solution that satisfies (4) if $\mu(t)$ a solution of the following integral equation

$$
\int_{0}^{t} K(t, \tau) \mu(\tau) d \tau=g(t)
$$

where

$$
\begin{aligned}
& K(t, \tau)=\frac{4 l_{1} l_{2}}{\pi^{4}} \sum_{n=1}^{\infty} \sum_{m=1}^{\infty} \frac{(-1)^{n+1}\left[1-(-1)^{n}\right]\left[1-(-1)^{m}\right]^{2}}{n^{2} m^{2}}\left(\lambda_{n m} a\right)^{2} e^{-\left(\lambda_{n m} a\right)^{2}(t-\tau)} \\
& g(t)=B-\frac{l_{1} l_{2}}{\pi^{2}} \sum_{n=1}^{\infty} \sum_{m=1}^{\infty} \frac{\left[1-(-1)^{n}\right]\left[1-(-1)^{m}\right]}{n m}\left[\hat{f}_{n m}(t)+c_{n m}\right] e^{-\left(\lambda_{n m} a\right)^{2} t} \\
& \hat{f}_{n m}(t)=\frac{4}{l_{1} l_{2}} \int_{0}^{t} \int_{0}^{l_{1}} \int_{0}^{l_{2}} f(\xi, \eta, \tau) \sin \frac{\pi n \xi}{l_{1}} \sin \frac{\pi m \eta}{l_{2}} e^{\left(\lambda_{n m} a\right)^{2} \tau} d \eta d \xi d \tau \\
& c_{n m}=\frac{4}{l_{1} l_{2}} \int_{0}^{l_{1}} \int_{0}^{l_{2}} \varphi(\xi, \eta) \sin \frac{\pi n \xi}{l_{1}} \sin \frac{\pi m \eta}{l_{2}} d \eta d \xi \\
& \lambda_{n m}=\sqrt{\left(\frac{\pi n}{l_{1}}\right)^{2}+\left(\frac{\pi m}{l_{2}}\right)^{2}} .
\end{aligned}
$$

Proof: We know that a solution of the problem (1), (3), (6) as follows

$$
u(x, y, t)=U(x, y, t)+\sum_{n=1}^{\infty} \sum_{m=1}^{\infty}\left[\bar{f}_{n m}(t)+c_{n m}\right] e^{-\left(\lambda_{n m} a\right)^{2} t} \sin \alpha_{n} x \cdot \sin \beta_{m} y
$$

where

$$
\begin{aligned}
& U(x, y, t)=\left\{\begin{array}{l}
\frac{x}{l_{1}} \mu(t) \quad \text { if } \quad 0<y<l_{2} \\
0 \quad \text { if } \quad y=0 \quad \text { and } \quad y=l_{2}
\end{array}\right. \\
& \bar{f}_{n m}(t)=\frac{4}{l_{1} l_{2}} \int_{0}^{t} \int_{0}^{l_{1} l_{2}} \int_{0}\left[f(\xi, \eta, \tau)-\frac{\xi}{l_{1}} \mu^{\prime}(\tau)\right] \sin \alpha_{n} \xi \cdot \sin \beta_{m} \eta \cdot e^{\lambda_{n m}^{2} a^{2} \tau} d \eta d \xi d \tau .
\end{aligned}
$$

According to the conditions of theorem 3, a solution (17) must satisfy (4) which means 


$$
\begin{aligned}
& B=\int_{0}^{l_{1}} \int_{0}^{l_{2}} u(x, y, t) d y d x=\int_{0}^{l_{1}} \int_{0}^{l_{2}} \frac{x}{l_{1}} \mu(t) d y d x+ \\
& +\int_{0}^{l_{1}} \int_{0}^{l_{2}} \sum_{n=1}^{\infty} \sum_{m=1}^{\infty}\left[\bar{f}_{n m}(t)+c_{n m}\right] e^{-\left(\lambda_{n m} a\right)^{2} t} \sin \alpha_{n} x \cdot \sin \beta_{m} y d y d x .
\end{aligned}
$$

In the second term, change the order of summation and integration after evaluation of the integrals to obtain the following

$$
B=\frac{l_{1} l_{2}}{2} \mu(t)+\frac{l_{1} l_{2}}{\pi^{2}} \sum_{n=1}^{\infty} \sum_{m=1}^{\infty}\left[\bar{f}_{n m}(t)+c_{n m}\right] e^{-\left(\lambda_{n m} a\right)^{2} t} \frac{1-(-1)^{n}}{n} \frac{1-(-1)^{m}}{m} .
$$

From (18) and (8), obtain

$$
\begin{aligned}
& B=\frac{l_{1} l_{2}}{2} \mu(t)+\frac{l_{1} l_{2}}{\pi^{2}} \sum_{n=1}^{\infty} \sum_{m=1}^{\infty}\left[\hat{f}_{n m}(t)+c_{n m}\right] e^{-\left(\lambda_{n m} a\right)^{2} t} \frac{\left[1-(-1)^{n}\right]\left[1-(-1)^{m}\right]}{n m}- \\
& -\frac{4}{\pi^{2}} \sum_{n=1}^{\infty} \sum_{m=1}^{\infty}\left[\int_{0}^{t} \int_{0}^{l_{1} l_{2}} \frac{x}{l_{1}} \mu^{\prime}(\tau) e^{-\left(\lambda_{n m} a\right)^{2}(t-\tau)} \sin \alpha_{n} x \cdot \sin \beta_{m} y d y d x d \tau\right] \times \\
& \times \frac{\left[1-(-1)^{n}\right]\left[1-(-1)^{m}\right]}{n m} .
\end{aligned}
$$

Taking into account

$$
\begin{aligned}
& \int_{0}^{t} \mu^{\prime}(\tau) e^{\left(\lambda_{n m} a\right)^{2} \tau} d \tau=\mu(t) e^{\left(\lambda_{n m} a\right)^{2} t}-\left(\lambda_{n m} a\right)^{2} \int_{0}^{t} \mu(\tau) e^{\left(\lambda_{n m} a\right)^{2} \tau} d \tau, \\
& \int_{0}^{l_{2}} \sin \beta_{m} y d y=\frac{l_{2}}{\pi m}\left[1-(-1)^{m}\right], \quad \int_{0}^{l_{1}} \frac{x}{l_{1}} \sin \alpha_{n} x d x=\frac{l_{1}}{\pi n}(-1)^{n+1} .
\end{aligned}
$$

From the last equality, by introducing the notation

$$
G(t)=\frac{l_{1} l_{2}}{\pi^{2}} \sum_{n=1}^{\infty} \sum_{m=1}^{\infty}\left[\hat{f}_{n m}(t)+c_{n m}\right] e^{-\left(\lambda_{n m} a\right)^{2} t} \frac{\left[1-(-1)^{n}\right]\left[1-(-1)^{m}\right]}{n m} .
$$

Obtain the following equation

$$
\begin{aligned}
& B=\frac{l_{1} l_{2}}{2} \mu(t)+G(t)- \\
& -\frac{4 l_{1} l_{2}}{\pi^{4}} \mu(t) \sum_{n=1}^{\infty} \sum_{m=1}^{\infty} \frac{(-1)^{n+1}\left[1-(-1)^{n}\right]\left[1-(-1)^{m}\right]^{2}}{n^{2} m^{2}}+ \\
& +\int_{0}^{t} \mu(\tau)\left\{\frac{4 l_{1} l_{2}}{\pi^{4}} \sum_{n=1}^{\infty} \sum_{m=1}^{\infty} \frac{(-1)^{n+1}\left[1-(-1)^{n}\right]\left[1-(-1)^{m}\right]^{2}}{n^{2} m^{2}}\left(\lambda_{n m} a\right)^{2} e^{-\left(\lambda_{n m} a\right)^{2}(t-\tau)}\right\} d \tau .
\end{aligned}
$$

By evaluation of the series in the third term and by denoting 


$$
K(t, \tau)=\frac{4 l_{1} l_{2}}{\pi^{4}} \sum_{n=1}^{\infty} \sum_{m=1}^{\infty} \frac{(-1)^{n+1}\left[1-(-1)^{n}\right]\left[1-(-1)^{m}\right]^{2}}{n^{2} m^{2}}\left(\lambda_{n m} a\right)^{2} e^{-\left(\lambda_{n m} a\right)^{2}(t-\tau)}
$$

obtain

$$
\int_{0}^{t} K(t, \tau) \mu(\tau) d \tau=B-G(t)
$$

If we denote $g(t)=B-G(t)$ then we obtain integral equation (16). The theorem is proven.

\section{CONCLUSION}

The results of this study have been analyzed and it can be concluded that both a fixed average amount of heat and a fixed heat at a given internal point of the rectangular plate can be controlled from the one of the boundaries. In this paper, a control function must be a solution of the integral equations found in this study.

\section{REFERENCES}

[1] Tikhonov AN, Samarsky AA. (1966) Equations of Mathematical Physics. Nauka, Moscow (in Russian)

[2] Lions JL. (1968) Contrôle Optimal de Systèmes Gouvernés par des Équations aux Dérivées Partielles. Dunod Gauthier-Villars, Paris

[3] Il'in VA. (2000) Differential equations. 36:1670-1686

[4] Il'in VA, Moiseev EI. (2005) Progress Math. Scien., 60(6):89-114. (in Russian).

[5] Il'in VA, Moiseev EI. (2006) Doc. Acad. Scien. Russian. 411(6):736-740. (in Russian).

[6] Fattorini HO. (2002) Time and norm optimal control for linear parabolic equations: necessary and sufficient conditions. In: Control and Estimation of Distributed Parameter Systems. International Series of Numerical Mathematics, 143:151-168. Birkhäuser, Basel

[7] Barbu V, Rascanu A, Tessitore G. (2003) Carleman estimates and controllability of linear stochastic heat equations. Appl. Math. Optim., 47(2):97-120.

[8] Alimov ShO. (2005) On a control problem associated with the Heat Exchange Process, Uz. Math. Jur. 4:13-21, (in Russian).

[9] Alimov ShO. (2008) On a control problem associated with the Heat Exchange Process. Doc. Acad. Scien. Russian, 421(5):583-585, (in Russian).

[10] Alimov ShO, Albeverio S. (2008) On a Time-Optimal Control Problem Associated with the Heat Exchange Process, Appl. Math. Optim. 57: 58-68.

[11] Alimov ShO. (2010) On a control problem associated with the heat transfer process, Eurasian mathematical journal, 1(2):17- 30.

[12] Alimov ShO. (2011) On the null-controllability of the heat exchange process, Eurasian mathematical journal, 2(3):5- 19.

[13] Fayziev YuE, Khalilova N. (2016) On a control problem associated with the heat transfer process, Вестник НУУ, 2/1:49-54, (in Russian). 